\title{
A Comparative Study of Methods for Separating Audio Signals
}

\section{Riham J. Issa}

rihamjassim11@gmail.com

College of Computer Sciences and Mathematics

Yusra F. Al-Irhaym

yusrafaisalcs@gmail.com

University of Mosul, Mosul, Iraq

Received on: 14/01/2020

Accepted on: 28/03/2020

\section{ABSTRACT}

The process of separating signal from a mixture of signals represents an essential task for many applications including sound signal processing and speech processing systems as well as medical signal processing. In this paper, a review of sound source separation problem has been presented, as well as the methods used to extract features from the audio signal, also, we define the Blind source separation problem and comparing between some of the methods used to solve the problem of source separation.

Keywords: Blind Source Separation, Independent Component Analysis, Deep Neural Networks.

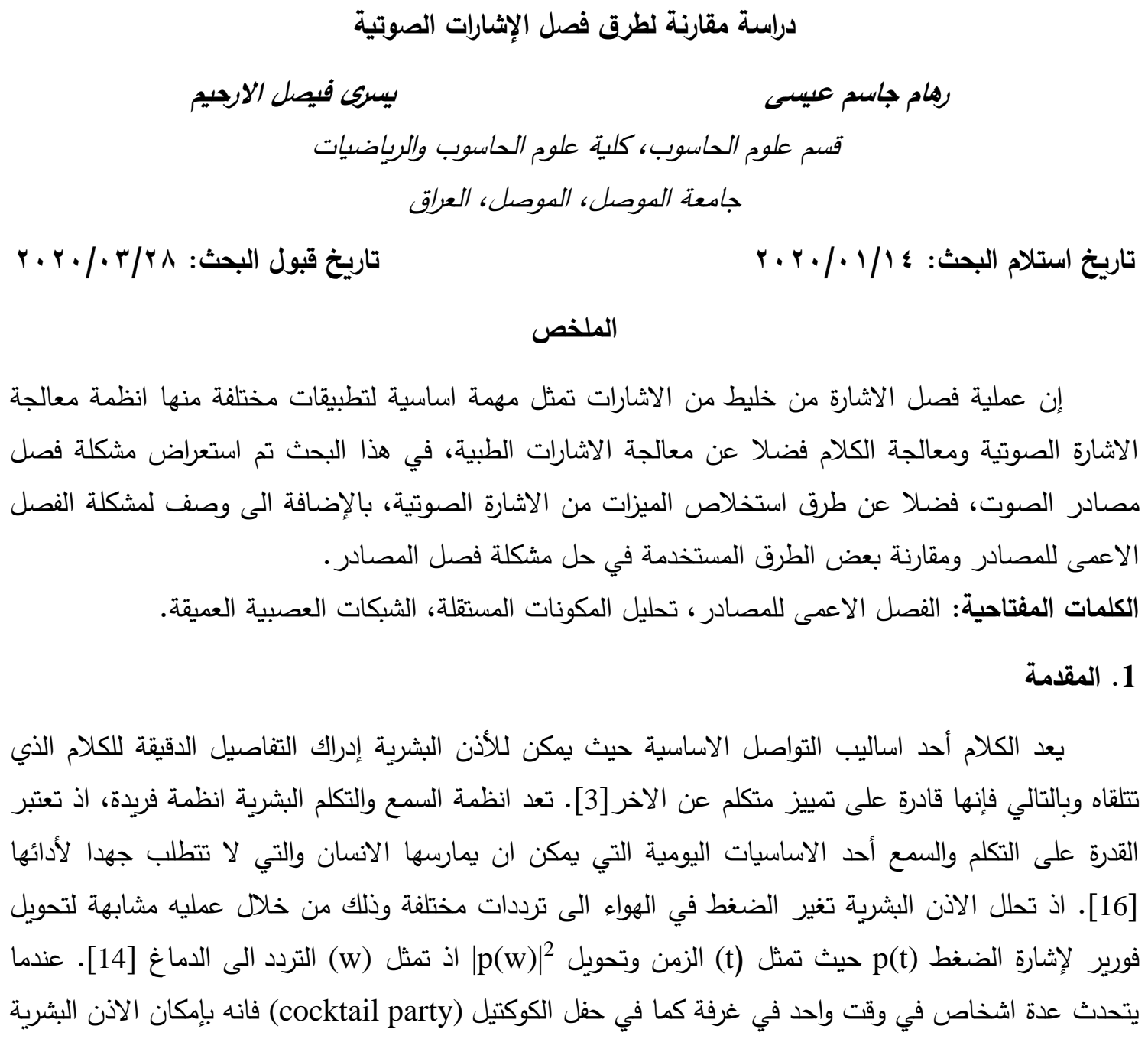$$
\text { دراسة مقارنة لطرق فصل الإثارات الصوتية }
$$

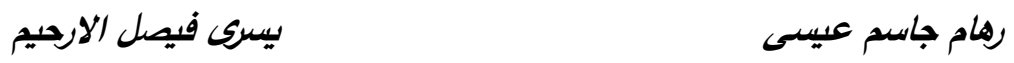$$
\text { قسم علوم الحاسوب، كلية علوم الحاسوب والرياضيات }
$$

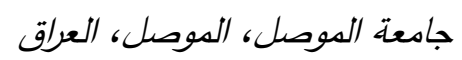

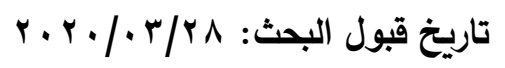

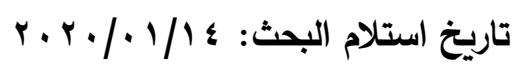

الملخص

إن عملية فصل الاشارة من خليط من الاشارات تمثل مهمة اساسية لتطبيقات مختلفة منها انظمة معالجة

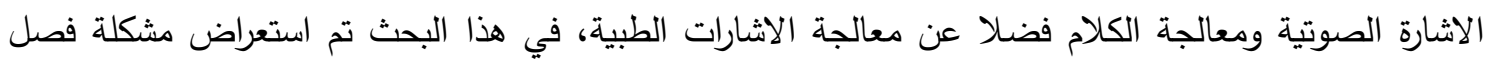

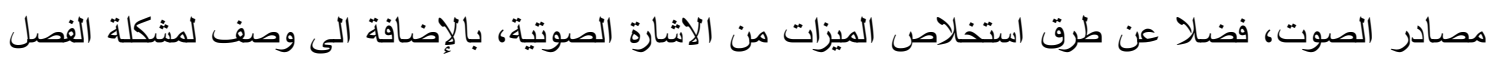

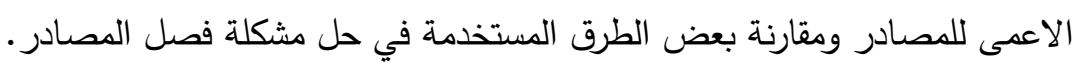
الكلمات المفتاحية: الفصل الاعمى للمصادر، تحليل المكونات المستقلة، الشبكات العصبية العميقة.

1

يعد الكلام أحد اساليب التواصل الاساسية حيث يمكن للأذن البشرية إدراك التفاصيل الدقيقة للكلام الذي

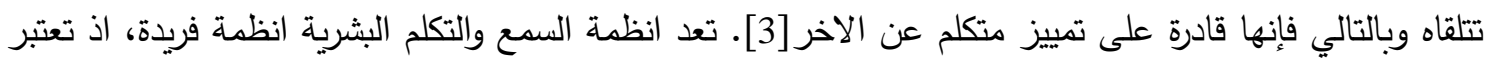

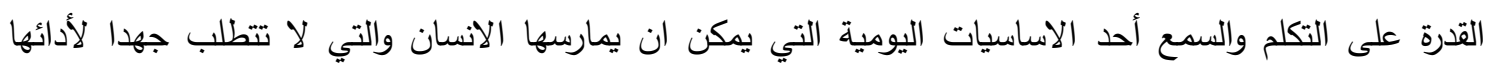
[16]. اذ تحلل الاذن البشرية تغير الضغط في الهواء الى ترددات مختلفة وذلك من خلال عمليه مشابهة لتحويل

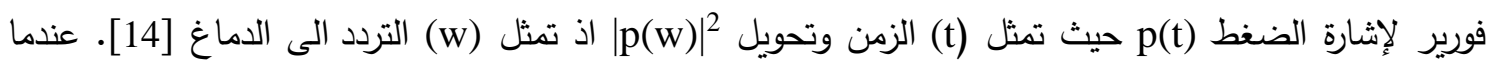
يتحدث عدة اشخاص في وقت واحد في غرفة كما في حفل الكوكتيل (cocktail party) فانه بإمكان الاذن البشرية 
الاصغاء الى مزيج من الاشارات مرغوبة واخرى غير مرغوبة ، ونظرا لما يتمتع به الانسان من ذكاء فانه بإمكانه تحديد مصادر الصوت النشطة فضلا عن قدرته على التركيز على مصدر صوت واحد وتجاهل بقية الاصوات باعتبارها ضوضاء الخلفية [1]. الا ان المعالجة الحاسوبية للإثشارات الصوتية في العديد من تطبيقات معالجة الاشارة تععل على تحليل ومعالجة الاشارة المعزولة بدقة أكثر من معالجة اشارة مكونة من خليط من الاشارات

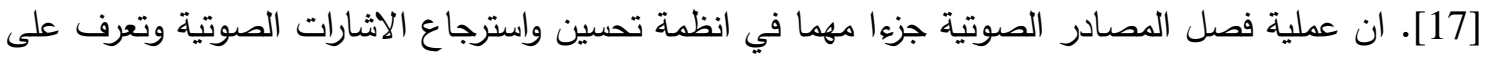
انها عملية استخراج مصادر الاشارات الصوتية الموجودة في خليط من الاشارات الصوتية [28]. وهناك العديد من الطرق لحل مشكلة فصل مصادر الصوت سواء كان التسجيل الصوتي من قناة واحدة او متعدد القنوات[12]. ونظرا لتطور حقل الثبكات الاصطناعية والتعلم العميق، ظهرت عدة معماريات لأنظمة التعلم لغرض فصل فئ فئ مصادر الصوت من قناة واحدة [25]، حيث اظهرت الثبكات الاصطناعية العميقة تميزا في الاداء في مهام فصل فلطئل المصادر كما في فصل الصوت المتكلم وفصل الصوت الموسيقى [27].

2. استخلاص الميزات (Feature Extraction)

يعرف استخلاص الميزات على انه عملية تحديد معلومات وخصائص ثابتة وكافية في الاشارة

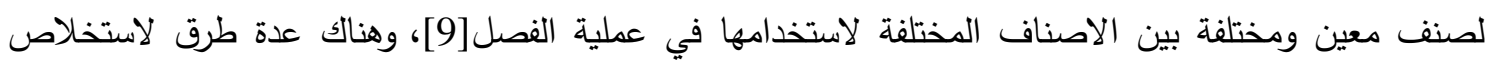

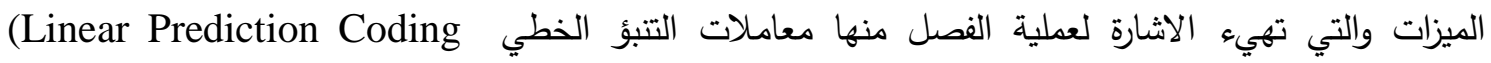

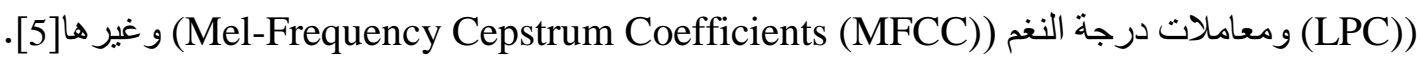

1.1 العبورات الصفرية (Zero Crossings)

تعتبر احد ابسط طرق استخلاص الميزات في الاشارة الصوتية والتي تستخدم عادة في تطبيقات التعرف على الكلام. حيث يحدث العبور الصفري في اشارات الزمن المتقطع في حالة كون العينات المتعاقبة ذات اشتارات جبرية فئية مختلفة، بينما يمثل معدل العبور الصفري مقياسا لعدد مرات التي تمر سعة اشارة الكلام خلال قيمة الصفر في فترة فئرة زمنية محددة [2].

2.2 تحويل فورير للوقت القصير (Short Time Fourier Transform (STFT) تستخدم هذه الطريقة لاستخلاص الميزات الزمنية والطيفية من خلال تحويل اشارة الصوت من المجال الزمني الى المجال الترددي، تحتوي الـ(STFT) على مجموعة من الباراميترات التي من الممكن ان تؤثر على حجم ودقة

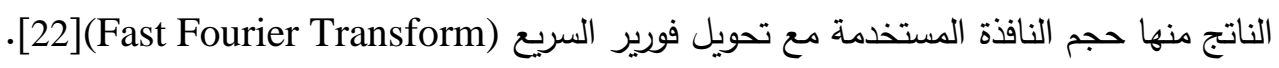

2.3 معاملات درجة النغم (Mel-Frequency Cepstrum Coefficients (MFCC))

تمثل الـ(MFCC) أحد الطرق الاكثر شيوعا في استخلاص ميزات الصوت في مهام التعرف على الكلام فهي تستند الى الادراك البشري للسمع في استخلاص الميزات، اذ تمثل القنوات الصوتية التي تتنج الكلام

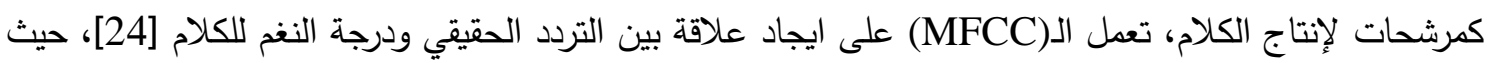

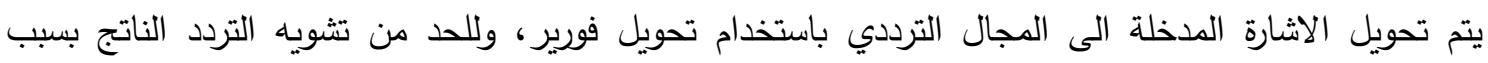
التجزئة يتم استخدام نافذة التحليل (Hammimg) قبل تطبيق تحويل فويرير • ومن ثم تحويل التردد من مقياس

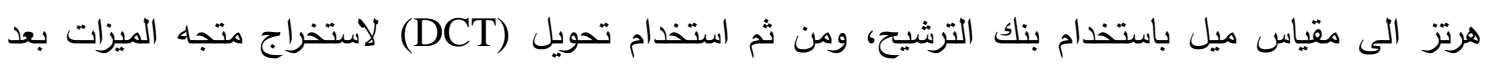


حساب اللوغارتم لقوة الطيف [5]. والثكل (1) يوضح الخطوات الاساسية لاستخلاص الميزات من الاشارة الصوتية باستخدام (MFCC). (M). (1).

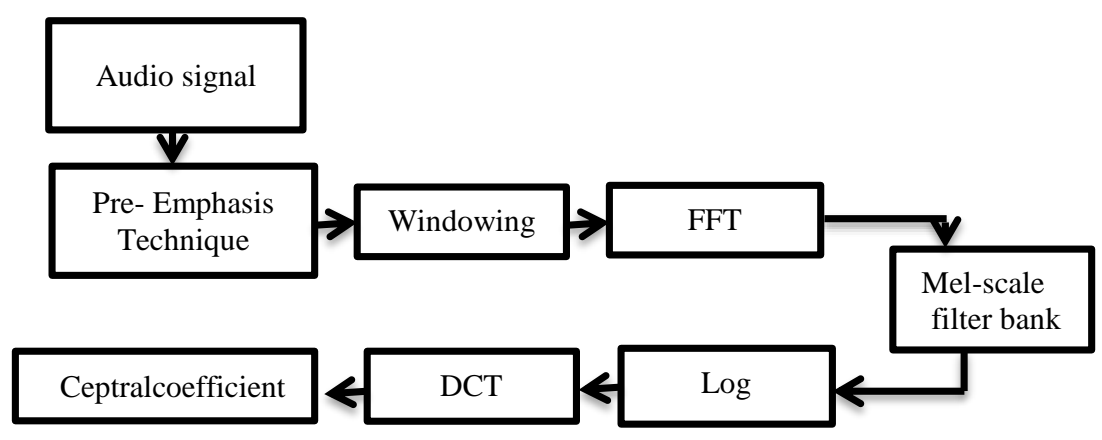

[24] الشكل (1) مخطط لمراحل الاشارة باستخدام (MFCC)

3. الفصل الاعمى للمصادر (Blind Source Separation)

تهذف عملية فصل المصادر الى استرجاع الاثارات الاصلية من خليط من الاشارات، والمثال الاكثر

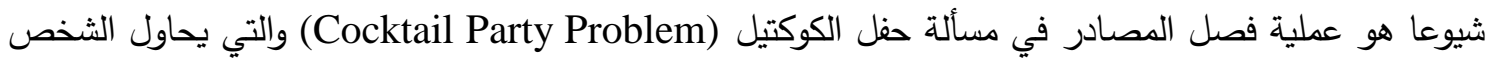
المستمع الى اتباع صوت متكلم محدد من بين مجموعة من اصوات للمتكلمين في غرفة واحدة وفي نفس الوقت[17]

3.1 تحليل المكونات المستقلة (Independent Component Analysis (ICA))

تمثل الـ(ICA) أحد الطرق الكلاسيكية واكثرها شهرة في مجال فصل الاشارات من مزيج خطي من مكونات

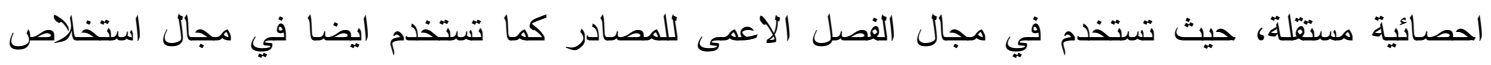
الميزات [1]. وتتطلب المعادلة القياسية لا (ICA) ان يكون عدد اشارات المصدر لا يتجاوز عدد الاشارات، فاذا كان عدد اشارات المصادر أكبر من عدد الاشارات يعرف بالمزيج الناقص او غير المكتمل[13]. كما ان اختلاف الرئيسي بين الـ(ICA) وطرق الـ(BSS) في البيانات (مستمرة، متقطعة) اضافة الى عشوائية المتغيرات، فالمكونات

في (ICA) يجب ان تكون مستقلة بينما في (BSS) فان الاستقلالية غير ضرورية [4].

3.2 الشبكات العصبية العميقة (Deep Neural Networks)

تستخدم الثبكات العصبية العميقة عناصر بسيطة مستوحاة من الانظمة العصبية البيولوجية لجمع بين طبقات معالجة لا خطية، فهي تتكون من طبقة ادخال وعدد من الطبقات المخفية وطبقة اخراج [8]، وتعد الثبكات

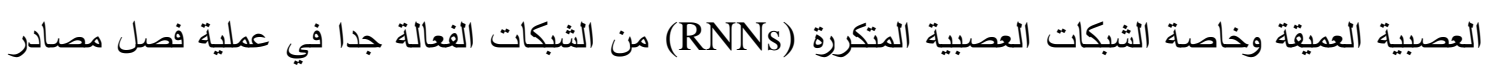
الصوت في حال توفر مجموعة بيانات كبيرة خاضعة للإشراف لتحسين اوزان الشبكة [23]. في هذا الجزء سيتم ايجاز انواع شبكات التعلم العميق الخاضع للإشراف وهي شبكة التغذية الامامية متعددة الطبقات (Feed-Forward Multilayer Perceptron's (MLPs))، والثبكة العصبية الالتفافية (Convolutional Neural Networks (CNNs)) وبالثبكات التوليدية التنافسية (Generative Adversarial Networks (GANs) Networks (RNNs)) 
3.2.1 الشبكة العصبية ذات التغذية الامامية ( Feed-Forward Neural Networks(FNN)

تستخدم الشبكة العصبية القياسية ذات التغذية الامامية والمتصلة كليا (FNN) لتخمين طيف المصدر، ولغرض استغلال السياقات الزمنية، تستخدم عدد نوافد متسلسلة كإدخال[18]. ويعد نموذج شبكة التغذية الامامية

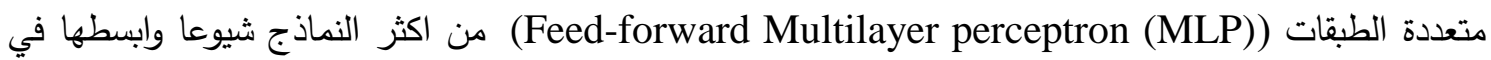
نماذج التعلم العميق التقليدية، حيث تكون الخلايا العصبية الموجودة في الطبقة li متصلة بجميع الخلايا في الطبقة

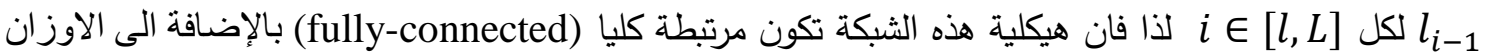

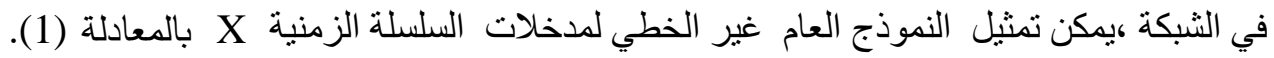
$A_{l i}=f\left(w_{l i} * X+b\right)$

حيث يمثل wli مجموعة الاوزان مع طول وعدد الابعاد التي تطابق المدخلات X’s ، بينما تمثل b الوزن التحيزي، اما عملية التعلم لثبكة الـ(MLP) هي المعالجة التي تكيف الارتباطات الموزونة لغرض الحصول على اقل فرق ما بين اخراج الثبكة والهدف وغالبا ما يتم استخدام خوارزميات الانتشار الخلفي (Back Propagation) التي تعتمد على تقانات تدرج النسب[10]. ولغرض زيادة كفاءة تدريب شبكات (MLP) يتم استخدام تقانات

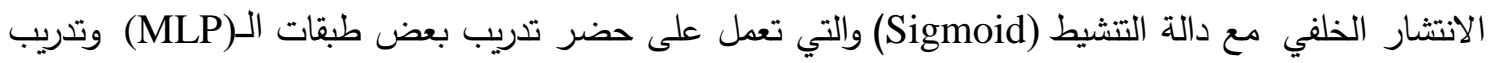
الطبقات المتبقية في نفس الوقت وذلك لتجنب ما يسمى بظاهرة التلاشي التدريجي (Vanishing Gradient) Problem) السفلى تبدا بالتتاقص او التلاشي تدريجيا، ونتيجة لهذا التلاشي فان الاوزان في الطبقات السفلى لا تعدل اوزانها كثيرا وهذا يؤدي الى ضعف عملية التعلم في الطبقات السفلية خلال عملية التدريب[7].

3.2.2 الثبكة العصبية الالتفافية (Convolutional Neural Network (CNN))

يعتمد هذا النوع من الثبكات العصبية على التفاف (convolving) مدخلات الثبكة مع نواة قابلة للتعلم. حيث ان الادخال لدالة التتشيط يتمثل بناتج التقاف الادخال الى الطبقة ومجموعة من الباراميترات (w) الخاصة بالطبقة والتي تسمى بالمرشحات او النواة. يتم في الطبقة الالتفافية (Convolutional Layer) حساب قنوات الات لهات ميزات متعددة قناة من النواة المقابلة لها، اما طبقات التجميع (Pooling Layers) فتستخدم لتبسيط قنوات الميزات المعلمة، وعادة ما تضاف هذه الطبقات فوق الطبقات الالتفافية، ففي الحالات ثنائية الابعاد فان الالتقاف الرياضي

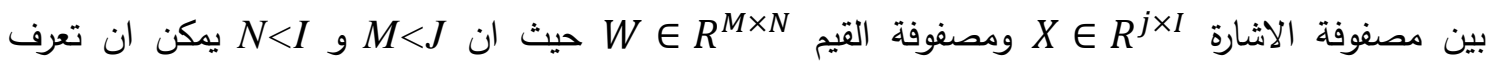

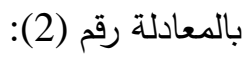

$S_{j . i}=\sum_{m=1}^{M} \sum_{n=1}^{N} x_{j, i}+m, i+n \omega_{m n}$

حيث تمثل كل من x,i و $x_{j, i} \omega_{m n}$ عدد الباراميترات بثكل كبير كما تحقق التعميم من خلال تبادل الباراميترات لنموذج الانماط المحلي في

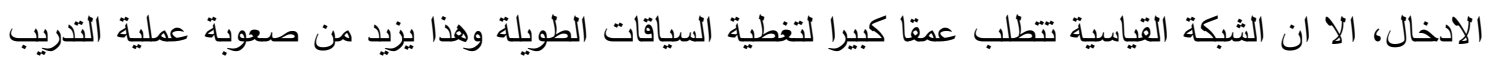
[18]. وعادة ما يتم تدريب شبكة ال(CNN) باستخدام خوارزمية الانتشار الخلفي الا انها تقلل من مشكلة التلاشي مني التدريجي، كما ان الخوارزمية المستندة الى التدرجات تدرب الثبكة بثكل كامل لتقليل معيار الخطأ بصورة مباشرة يجعل هذا النوع من الثبكات قادرا على تحقيق اوزان مثلى [15]. 
(Recurrent Neural Networks (RNNs)) 3.2.3 الثبكة العصبية التكرارية

تعد الشبكة العصبية التكرارية واحدة من اولى الثبكات القادرة على تذكر مدخلاتها، لأنها تتضمن ذاكرة

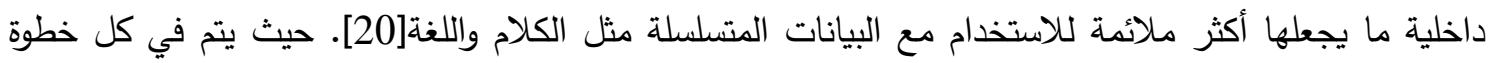
زمنية ارسال المدخلات من خلال الثبكة التكرارية، حيث تستلم العقد المدخلات عبر الحواف متكررة، بينما تستلم مدخلات التتشيط من متجه المدخلات الحالي ومن العقد المخفية في الحالة السابقة للثبكة، و يتم حساب الاخراج من الحالة المخفية في الخطوة الزمنية الحالية، ومن خلال الربط المتكرر فان متجه الادخال السابق في الخطوة الزمنية السابقة يؤثر على ناتج الاخراج الحالي في الخطوة الزمنية الحالية[8]. ويمكن تمثيل الثبكة التكرارية ذات الطبقة الواحدة بالمعادلة(3):

$h^{(n)}=\phi\left(W x^{(n)}+V h^{(n-1)}+b\right.$

وتمثل (.) و دالة التتشيط بينما b فهي المتجه التحيزي، اما V و W فتمثل قيم المصفوفات بينما اخراج الثبكة في الزمن n [16]. ولغرض التخلص من مشكلة التلاشي التدريجي، تستخدم معماريات مختلفة لثبكات ال(RNN) تدعى بـ (Gated-RNNs) ومن هذه المعماريات ما يسمى بـ (Long Short-Term)

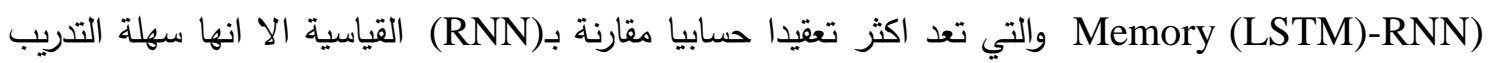
وتعمل بشكل افضل اذا كانت (N) كبيرة. كما تعد هذه البنية هي أكثر المعماريات شيوعا والتي تستخدم في دئي

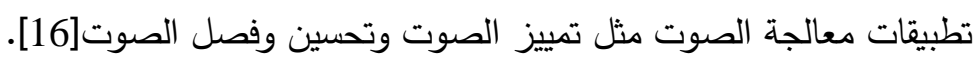

3.3.3 الثبكات الخصومية (Generative Adversarial Networks (GANs)

في السنوات الاخيرة قدمت هذه الثبكة لحل مشكلة فصل المصادر، حيث يفرض في هذه الطرق ان مرشحات المزج في اشارة من القناة الواحدة معلومة[21]، وتمثل هذه الثبكات اطارا لشبكتين فرعيتين وهما (Generator and Discriminator(G and D)) التوليد الـGenerator) لإنتاج عينات من هدف موزع معين. ولتحقيق ذلك، تستخدم شبكة التمييز

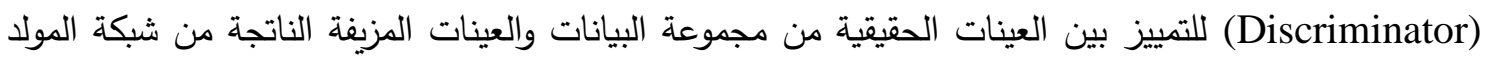

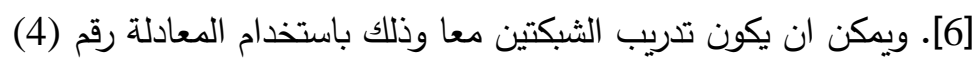
$\min _{G}^{\max } V_{C G A N}(G, D)=E_{x, \sim P_{\text {data(x) }}}[\log D(x)]+$

$$
E_{z} \sim P_{G(z)}[\log (1-D(G(z))]
$$

اذ تمثل x عينات بيانات حقيقية تم اخذها من التوزيع

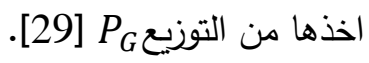

4. ق قواعد البيانات المستخدمة في عملية الفصل

تتكون قاعدة البيانات من 1000 مقطع غنائي بأصوات مختلفة (ذكر_انثى) تتراوح مدتها ما بين 4-13 ثانية ومعدل عينة 16,000 هرتز. اما قاعدة البيانات (DSD100) فتتضمن 100 مقطع موسيقي بتردد عينة

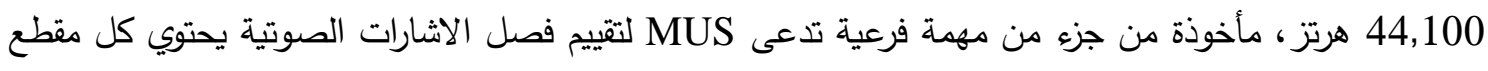

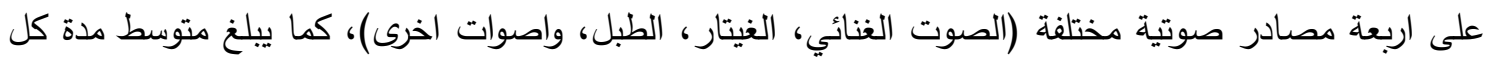
مقطع موسيقي 4 دقائق و 10 ثوان [29]. 
5. مقارنة نتائج طرق الفصل باستخدام الثبكات العصبية العميقة

إن عمليات فصل الصوت باستخدام قناة واحدة وباستخدام معماريات مختلفة للشبكات العصبية العميقة

اصبحت موضع اهتمام العديد من الباحثين، حيث قدمت العديد من الطرق لتحقيق افضل نتائج في عمليات الفصل، وفي هذا الجزء سيتم مناقشة نتائج فصل مصادر الصوت الغنائي عن الخلفية الموسيقية باستخدام الثبكات العصبية العميقة لثلاث شبكات عصبية وهي (SVSGAN) و و(MMDenseLSTM) و Stacked) (Signal-to-Distortion Ratio وفقا لبعض المقاييس وهي نسبة الاشارة الى التشوه Hourglass Model) ونسبة الاثارة الى التداخل (SDR))

عملية الفصل بصورة عامة (Signal to Artifacts Ratio (SAR). ومن ابرز الهيكليات المستخدمة في فصل الصوت الغنائي عن الخلفية الموسيقية باستخدام قناة واحدة هي

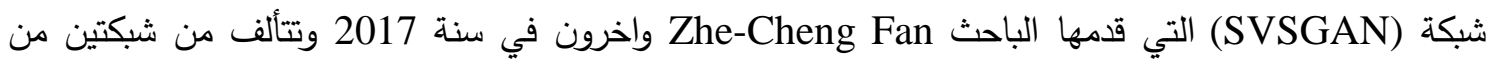
الثبكات العصبية التقليدية العميقة، الاولى شبكة التوليد G مدخلاتها اطياف الخليط اذ تعمل على توليد اطياف الصوت الغنائي واطياف الموسيقى الخلفية والثانية شبكة التوليف D التي تعمل على تمييز الصوت النظيف من

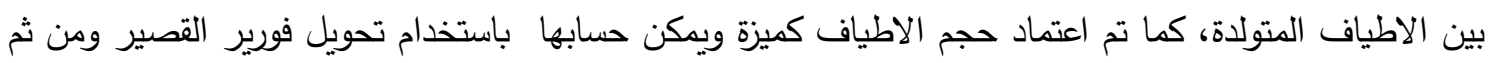
استخدام مرشح التردد الزمني (Ideal Ratio Mask IRM) لتحسين ناتج عملية الفصل، وقد تم استخدام شبكة

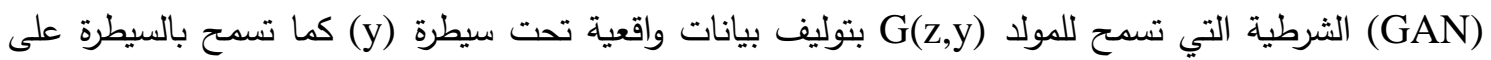
ناتج النموذج التوليفي D المتمثل بـ D(x,y) بواسطة متجه السياق (y) كما في معادلة رقم (5) التي تمثل دالة الهدف، واظهرت النتائج ان استخدام هذه الهيكلية قد حقق تحسينا في اداء عملية الفصل [29]. $\min _{G}^{\max } V_{c G A N}(G, D)=E_{z, s_{C} \sim P_{\operatorname{data}\left(z, s_{C}\right)}}\left[\log D\left(s_{c}, z\right)\right]+$

$$
E_{z} \sim P_{G(z)}[\log (1-D(G(z), z))]
$$

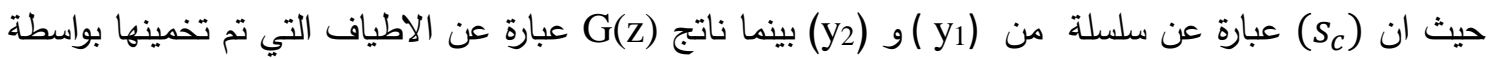
الا طياف المدخلة (z) اما شبكة التوليف (D) فيتم السيطرة عليها بواسطة قيم المتغيرات لأطياف الادخال (z). بينما قدم الباحث Naoya Takahashi واخرون في سنة 2018 طريقة تهدف لفصل الصوت من خلال دمج الثبكة العصبية الالتفافية والثبكة العصبية التكرارية باستخدام معماريتين مختلفتين وهما معمارية الـ -Multi) scale Multi- Band DenseNet(MMDenseNet)) حيث تم استخدام تحويل فورير القصير للحصول على حجم الطيف (Long short term memory (LSTM)) لإثارة الخليط، وقد تم تدريب الثبكة لتخمين طيف المصدر وذلك من خلال تقليل نسبة متوسط مربع الخطأ وقد اظهرت هذه الطريقة ان استخدام معمارية (Mean square Error MSE) من قلة عدد الباراميترات المستخدمة تفوقت على نوع اخر من الثبكات المدمجة وهو (MMDenseBLSTM)، كما تفوقت ايضا عن استخدام المرشح الثائي (Ideal Binary Mask (IBM)) في فصل الصوت الغنائي عن الموسيقى في شبكة مدربة على 900 مقطع غنائي[18]. نوع اخر من الثبكات العصبية قدمها الباحث Sungheon Park واخرون في 2018 وهي الثبكة العصبية الالتفافية وبالأخص هيكلية (Stacked hourglass network) في فصل مصدر الموسيقى، وهي شبكة مشابها لثبكة الـ(U-Net) حيث تم في هذه الطريقة اعتماد طيف الصورة مختلف الابعاد لتوليد مرشح لكل مصدر 
موسيقي ومن ثم تحسين المرشح الذي تم تخمينه من خلال تمريره في (stacked hourglass model). حيث يمكن وباستخدام شبكة واحدة فصل العديد من المصادر الموسيقية وقد حققت هذه الطريقة نتائج تنافسية قابلة للمقارنة مع احدث اساليب فصل الاصوات الموسيقية المتعددة ومهام فصل الصوت الغنائي[27]، والجدول (1) يوضح الفرق بين المقاييس باستخدام الشبكات الثلاث وباستخدام قواعد بيانات مختلفة.

جدول (1) نتائج (SIR-SDR-SAR) للشبكات العصبية

\begin{tabular}{|c|c|c|c|c|c|c|}
\hline $\begin{array}{c}\text { SAR } \\
\text { db }\end{array}$ & $\begin{array}{c}\text { SIR } \\
\text { db }\end{array}$ & $\begin{array}{c}\text { SDR } \\
\text { db }\end{array}$ & الصوت & ق ق قاعدة البيانات & نوع الثبكة & المصدر \\
\hline 10.14 & 9.84 & 6.57 & Vocal+ background music & MIR-1K & DNN(Baseline ) & [29] \\
\hline 10.32 & 9.86 & 6.69 & Vocal+ background music & MIR-1K & (SVSGAN) & [29] \\
\hline \multirow{5}{*}{ - } & \multirow{5}{*}{ - } & 3.73 & Bass & \multirow{5}{*}{ DSD100 } & \multirow{5}{*}{ (MMDenseLSTM) } & \multirow{5}{*}{ [18] } \\
\hline & & 5.46 & Drums & & & \\
\hline & & 6.31 & Vocal & & & \\
\hline & & 4.33 & Others & & & \\
\hline & & 12.37 & Accompaniments & & & \\
\hline 12.53 & 16.01 & 10.51 & Singing voice & \multirow{2}{*}{ MIR-1K } & \multirow{6}{*}{$\begin{array}{c}4 \text { stacked hourglass } \\
\text { networks }\end{array}$} & \multirow{6}{*}{ [27] } \\
\hline 12.36 & 14.24 & 9.88 & Accompaniments & & & \\
\hline- & - & 1.77 & Bass & \multirow{4}{*}{ DSD100 } & & \\
\hline- & - & 4.11 & Drums & & & \\
\hline- & - & 5.16 & Vocals & & & \\
\hline- & - & 2.36 & Other & & & \\
\hline- & - & 3.91 & Bass & \multirow{5}{*}{ DSD100 } & \multirow{5}{*}{ MMDenseNet } & \multirow{5}{*}{ [19] } \\
\hline- & - & 5.37 & Drums & & & \\
\hline- & - & 6.00 & Vocals & & & \\
\hline \multirow[t]{2}{*}{-} & - & 3.81 & Other & & & \\
\hline & & 12.10 & Accompaniments & & & \\
\hline
\end{tabular}

من خلال الجدول (1) نلاحظ ان شبكة (SVSGAN) المستخدمة لفصل الصوت الغنائي عن الموسيقى وعلى فرض ان خليط الاثارة مكون من جزأين الاول هو الصوت والاخر هو الموسيقى، اظهرت تحسنا في اداء عملية الفصل حيث تم مقارنتها بالثبكة العصبية العميقة التقليدية تتكون من ثلاث طبقات مخفية بالإضافة الى هلى 1024 خلية في كل طبقة، يمكن ملاحظة ان تطبيق الطريقتين على قاعدة البيانات MIR-1K اظهرت زيادة نسبة الاشارة الى التثوه (SDR) باستخدام الـ (SVSGAN) بمقدار 0.12db، كما ازدادت نسبة الاشارة الى التداخل (SIR) بمقدار db 0.02 وهي نسبة قليلة وقد لا تكون واضحة، اما نسبة (SAR) فقد ازدادت بمقدار

اما نتائج تدريب شبكة (4-stacked hourglass network) باستخدام قاعدة البيانات MIR-1K ومن خلال حساب الوسيط لكل من المقاييس نجد ان فصل الصوت الغنائي حقق اعلى نسب في المقاييس الثلاث بينما الصوت الموسيقي فقد حقق اعلى نسب بمقياسي (SDR,SIR)، وبذلك فانه كلما ازداد عدد نماذج stacked

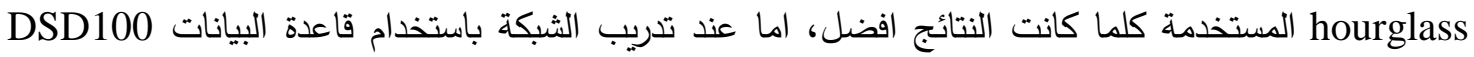

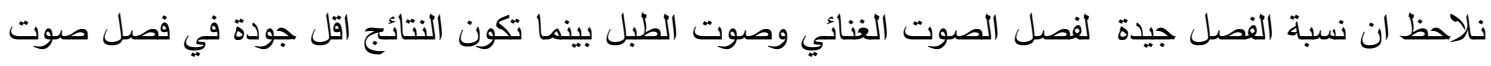
البيس كيتار وبقية الاصوات وذلك بسبب تقارب صوت البيس كيتار من صوت الكيتار العادي وبالتالي يؤثر في عملية التدريب عندما يتم تدريب الاصوات بنفس الثبكة[28-27]. اما شبكة (MMDenseLSTM) المستخدمة لفصل اصوات الادوات الموسيقية نجد ان الطريقة المقترحة تحقق تفوقا واضحا في فصل المصادر بالرغم من قلة

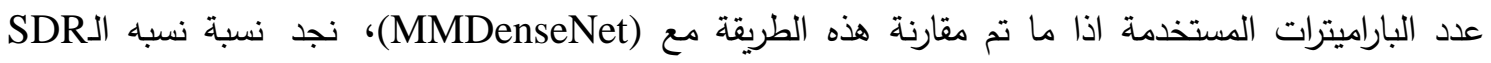


لاشارة الصوت الغنائي ازدادت بمقدار 0.31db عند استخدام MMdenseLSTM، كما ازدادت بمقدار بالنسبة لصوت الطبل اما بقية الاصوات الموسيقية 0.52db وهي نسبة عالية جدا، اما .Acco فكانت نسبة الفرق بمقدار 0.63db

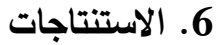

تختلف الطرق المستخدمة في عمليات فصل المصادر الصوتية في مدى قوتها في تحقيق عملية الفصل كما تختلف بمدى ملائمتها لإداء المهام المطلوبة، وفي مهمة فصل الاصوات الموسيقية، تم اجراء مقارنة لنتائج ثلاث طرق للفصل بالاعتماد على مقاييس اهمها نسبة التشوه ونسبة التداخل في الاشارة التي تم استرجاعها، وكما موضح في الجدول (1) فان استخدام الثبكة العصبية الالتفافية العميقة (4-stacked hourglass network) (المصممة لتخمين حالة الانسان في الصور الرقمية الملونة) لفصل الصوت الغنائي عن الموسيقي اعطت نتائج افضل من استخدام الثبكة العصبية (SVSGAN) حيث حقتت زيادة في النسب (SDR) بمقدار 3.82db)

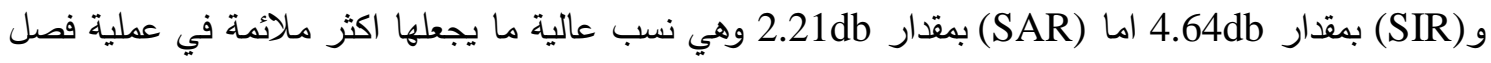
الصوت الغنائي عن الخلفية الموسيقية، اما في فصل اصوات الادوات الموسيقية نلاحظ ان الثبكة العصبية

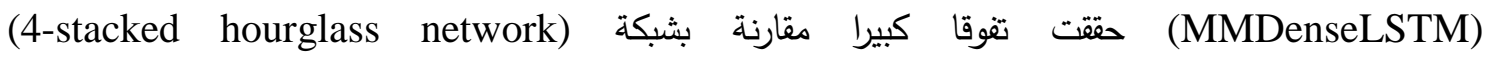

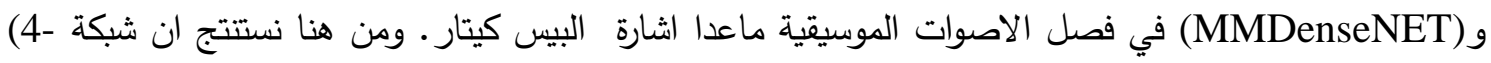
stacked hourglass network) فهي تحقق نتائج فصل أفضل بشكل عام من الطرق الاخرى. 


\section{المصادر}

[1] Abouzid Houda, Chakkor Otman, 2017, "A Novel Method Based on Gaussianity and Sparsity for Signal Separation Algorithms", International Journal of Electrical and Computer Engineering (IJECE), Vol.7, No.4.

[2] Bachu R.G., Kopparthi S., Adapa B., Barkana B.D., 2014, "Separation Of Voiced and Unvoiced Using Zero Crossing Rate And Energy of The Speech Signal", Bulletin of The Polish Academy of Sciences, Vol. 62, No. 3.

[3] Bhavana V.S, Pradip K. Das, 2019, "Speaker Verification Using Simple Temporal Features and Pitch Synchronous Cepstral Coefficients", Arxiv:1908.05553v1.

[4] Birmingham Hang Guan, Anand Rangarajan, 2018, "Signals as Parametric Curves: Application to Independent Component Analysis and Blind Source Separation", http://Arxiv.Org/Abs/1807.03442.

[5] C. Sunitha, E. Chandra, 2015, "Speaker Recognition Using Mfcc And Improved Weighted Vector Quantization Algorithm", International Journal Of Engineering and Technology (IJET), ISSN: 0975-4024, Vol. 7, No .5.

[6] Daniel Stoller Daniel, Sebastian Ewert, Simon Dixon, 2019, "Training Generative Adversarial Networks from Incomplete Observations Using Factorised Discriminators", International Conference on Learning Representations (ICLR), Arxiv:1905.12660v1.

[7] Deliang Wang, Jitong Chen, 2018, "Supervised Speech Separation Based on Deep Learning: An Overview , IEEE/ACM Transactions on Audio, Speech and Language Processing (TASLP), Vol. 26, No. 10.

[8] Faisal Mohammad, Ki-Boem Lee, Young-Chon Kim, 2018, "Short Term Load Forecasting Using Deep Neural Networks", International Symposium on Information Technology Convergence (ISITC), arXiv:1811.03242 .

[9] Hassan Ismail Fawaz, Germain Forestier, Jonathan Weber, Lhassane Idoumghar, Pierre-Alain Muller, 2018, "Deep Learning For Time Series Classification: A Review", Data Mining And Knowledge Discovery, Issue 4, https://Doi.Org/10.1007/S10618-019-00619-1 .

[10] Hassan Ramchoun, Mohammed Amine Janati Idrissi, Youssef Ghanou, Mohamed Ettaouil, 2016, "Multilayer Perceptron: Architecture Optimization and Training", International Journal of Interactive Multimedia and Artificial Intelligence, Vol. 4, No.1.

[11] Imad Rida, 2018, "Feature Extraction for Temporal Signal Recognition: An Overview", Computer Science, Engineering, Arxiv:1812.01780.

[12] Joonas Nikunen, Aleksandr Diment, Tuomas Virtanen, 2017, "Separation of Moving Sound Sources Using Multichannel NMF and Acoustic Tracking", IEEE/ACM Transactions On Audio, Speech, And Language Processing, Vol. 26, pp. 281-295. 
[13] K. Prakash, Hepzibha Rani D, 2015, "Blind Source Separation for Speech Music and Speech Speech Mixtures", International Journal of Computer Applications (0975 - 8887), Vol. 110, No. 12.

[14] Keith Y. Patarroyo, Vladimir Vargas Calderon, 2017, "Pronunciation Recognition of English Phonemes/Ә/,/Æ,/a:/And/^/Using Formants And Mel Frequency Cepstral Coefficients", Arxiv:1702.07071.

[15] M. Z. Alom, T. M. Taha, C. Yakopcic, S. Westberg, P. Sidike, M.S. Nasrin, M. Hasan, B.C. Van Essen, AA.S. Awwal, V.K. Asar, 2019, "A State-of-The-Art Survey on Deep Learning Theory and Architectures", MDPI Electronics (292), Vol. 8, No. 3.

[16] Morten Kolbaek, 2018, "Single-Microphone Speech Enhancement and Separation Using Deep Learning", Ph.D. Thesis, Aalborg University, Denmark.

[17] Ms. Monali R. Pimpale, Prof. Shanthi Therese, Prof. Vinayak Shinde, 2016, "A Survey On: Sound Source Separation Methods", International Journal of Computer Engineering in Research Trends, Vol. 3, pp. 580-584.

[18] Naoya Takahashi, Nabarun Goswami, Yuki Mitsufuji, 2018, "MMDENSELSTM: An Efficient Combination of Convolutional and Recurrent Neural Networks for Audio Source Separation", IEEE, 16th International Workshop on Acoustic Signal Enhancement (IWAENC), DOI: 10.1109/IWAENC.2018.8521383.

[19] Naoya Takahashi And Yuki Mitsufuji., 2017, "Multi-Scale Multi-Band Dense Nets for Audio Source Separation", In Applications of Signal Processing to Audio and Acoustics (Waspaa), IEEE Workshop On, pp. 21-25.

[20] Nilay Ganatra, Atul Patel, 2018, "A Comprehensive Study of Deep Learning Architectures, Applications and Tools", International Journal of Computer Sciences And Engineering, Vol. 6, Issue 12, E-ISSN: 2347-2693.

[21] Qiuqiang Kong, Yong Xu, Wenwu Wang, Philip J.B. Jackson and Mark D. Plumbley, 2019, "Single-Channel Signal Separation and Deconvolution With Generative Adversarial Networks", 28th International Joint Conference On Artificial Intelligence (IJCAI-19), DOI:10.24963/IJSAI.2019/381, pp.27472753.

[22] Saber Malekzadeh, Shahla Rezazadeh Azar, Maryam Samami, Maryam Rayegan, 2019, "Classical Music Generation In Distinct Dastgahs with Alimnet Acgan", 27th Iranian Conference on Electrical Engineering, Arxiv:1901.04696 .

[23] Scott Wisdom, Thomas Powers, James Pitton, Les Atlas, 2017, "Deep Recurrent Nmf for Speech Separation by Unfolding Iterative Thresholdin", IEEE Workshop On Applications of Signal Processing to Audio and Acoustics, Doi:10.1109/Waspaa.2017.8170034,E-ISSN: 1947-1629.

[24] Shaik Riyaz, Bathula Lakshmi Bhavani, S.Venkatrama Phani Kumar, 2019, "Automatic Speaker Recognition System in Urdu Using MFCC \& HMM", International Journal Of Recent Technology And Engineering (IJRTE), ISSN:2277-3878, Vol. 7, Issue 5S4. 
[25] Shrikant Venkataramani, Paris Smaragdis, 2018, "End-To-End Networks For Supervised Single-Channel Speech Separation", IEEE Journal of Selected Topics in Signal Processing (J-STSP), Arxiv:1810.02568.

[26] Steven Spratley, Daniel Beck, And Trevor Cohn, 2019, "A Unified Neural Architecture for Instrumental Audio Tasks", PIEEE, International Conference On Acoustics, Speech, and Signal Processing. DOI: $10.1109 /$ Icassp.2019.8682765.

[27] Sungheon Park, Taehoon Kim, Kyogu Lee, Nojun Kwak, 2018, "Music Source Separation Using Stacked Hourglass Networks", Computer Science, Engineering, ISMIR, Arxiv:1805.08559v2 .

[28] V.S. Narayanaswamy, S. Katoch, J. J. Thiagaraja, H. Song, A. Spanias, 2019, "Audio Source Separation Via Multi-Scale Learning With Dilated Dense UNets", Arxiv:1904.04161 .

[29] Zhe-Cheng Fan, Yen-Lin Lai, Jyh-Shing R. Jang, 2017, "SVSGAN: Singing Voice Separation Via Generative Adversarial Network", IEEE International Conference on Acoustics, Speech and Signal Processing (ICASSP), DOI: 10.1109/ICASSP.2018.8462091. 